hep-ph/0506309

\title{
Off-shell scattering amplitudes in the double-logarithmic approximation
}

\author{
B.I. Ermolaev \\ Ioffe Physico-Technical Institute, 194021 St.Petersburg, Russia \\ M. Greco \\ Department of Physics and INFN, University Rome III, Rome, Italy \\ F. Olness \\ Department of Physics, Southern Methodist University, Dallas, TX 75275-0175 USA \\ S.I. Troyan \\ St.Petersburg Institute of Nuclear Physics, 188300 Gatchina, Russia
}

\begin{abstract}
When scattering amplitudes are calculated in the double-logarithmic approximation, it is possible to relate the double-logarithmic on-shell and off-shell amplitudes. Explicit relations are obtained for scattering amplitudes in QED, QCD, and the ElectroWeak Standard Model. The off-shell amplitudes are considered in the hard and the Regge kinematic limits. We compare our results in both the Feynman and Coulomb gauges.

PACS numbers: 12.38.Cy
\end{abstract}

\section{INTRODUCTION}

Because off-shell scattering amplitudes are not gauge-invariant quantities, they have not been studied as extensively as their on-shell counterparts. Nevertheless, off-shell scattering amplitudes are important to calculate as they arise in many well-known cases, such as when the process of interest can be divided into sub-processes. One example is the $e^{+} e^{-}$annihilation into off-shell unstable particles (a quark pair, or a $W^{+} W^{-}$- pair) followed by the decay of the off-shell states. Other examples include the Bethe-Salpeter equation which relates off-shell amplitudes to onshell amplitudes, and the DGLAP 1] evolution equations which operate with off-shell amplitudes. The Deep-Inelastic Scattering (DIS) structure functions provide yet another example when the initial parton is not assumed to be on the mass shell.

In general, the expressions for the on-shell and off-shell scattering amplitudes are quite different and should be calculated independently. There exist certain cases where it is possible to relate off-shell and on-shell amplitudes; however, even if we limit our scope to the the double logarithmic approximation (DLA), there are many cases where the on-shell amplitude cannot be obtained by a simple limit of the off-shell amplitude.

In order to demonstrate the relation between the off-shell and on-shell amplitudes, let us consider the well-known example of the Sudakov form factor in QED. [2] When the virtualities of the initial $\left(p_{1}^{2}\right)$ and the final $\left(p_{2}^{2}\right)$ electrons are much greater than the electron mass squared $\left(\left|p_{1,2}^{2}\right| \gg m^{2}\right)$, the expression for the off-shell Sudakov form-factor $S\left(q^{2}\right)$ of electron in QED is:

$$
S\left(q^{2}\right)_{\text {off }}=\exp \left[-(\alpha / 2 \pi) \ln \left(-q^{2} /\left|p_{1}^{2}\right|\right) \ln \left(-q^{2} /\left|p_{2}^{2}\right|\right)\right]
$$

where $q$ is the momentum transfer: $q=p_{2}-p_{1}$. We note that Eq. (11) accounts for all DL contributions to the first part $\left(\sim \gamma_{\mu}\right)$ of the photon-electron vertex ${ }^{1}$ in the limit $\left|q^{2}\right| \gg\left|p_{1,2}^{2}\right|$. Conversely, when the electron is on-shell before and after the scattering process, the Sudakov form-factor is

$$
S\left(q^{2}\right)_{\text {on }}=\exp \left[-(\alpha / 4 \pi) \ln ^{2}\left(-q^{2} / m^{2}\right)\right] .
$$

The minus sign in the logarithmic terms $\ln \left(-q^{2}\right)$ of Eqs. (12) is related to the analyticity in the $q^{2}$-plane; indeed, the Sudakov form factor should not have an imaginary part when $q^{2}<0$. To simplify the notation, we will drop this minus sign, together with the modulus for $\left|p_{1,2}^{2}\right|$, throughout the paper.

\footnotetext{
1 The expressions for the DL asymptotics of the second part $\left(\sim \sigma_{\mu \nu}\right)$ of the photon-fermion vertex are obtained in Ref. [3]
} 
Obviously, Eq. (2) cannot be obtained from Eq. (1) by taking the limit $p_{1}^{2}=p_{2}^{2}=m^{2}$. Hence, the on-shell and off-shell Sudakov form-factors cannot be related by a simple analytic continuation of the mass scales. This result stems from the fact that the DL contributions to the Sudakov form-factors come from infrared singularities in the integration over the virtual photon momenta; the separate mass scales $m^{2}$ and $p_{1,2}^{2}$ regulate these singularities.

In the one-loop approximation, the general expression for the DL contribution to the Sudakov form-factors can be written as:

$$
W=-(\alpha / 4 \pi)\left[\ln ^{2}\left(q^{2} / m^{2}\right)-\ln ^{2}\left(p_{1}^{2} / m^{2}\right)-\ln ^{2}\left(p_{2}^{2} / m^{2}\right)+\Theta\left(p_{1}^{2} p_{2}^{2}-m^{2} q^{2}\right) \ln ^{2}\left(m^{2} q^{2} / p_{1}^{2} p_{2}^{2}\right)\right]
$$

where the value of the infrared cut-off $\mu$ is chosen as $(\mu \approx m)$. Summing the higher loop contributions for $W$ leads to the exponentiation of the one-loop result. The individual terms in the squared brackets in Eq. (3) are obtained from the integration over separate kinematic regions.

The calculation in DLA is especially simple when the Sudakov parametrization is used for the soft momenta $k_{\mu}$ :

$$
k_{\mu}=\alpha p_{2 \mu}^{\prime}+\beta p_{1 \mu}^{\prime}+k_{\perp \mu},
$$

with $p^{\prime}{ }_{1 \mu}=p_{1 \mu}-\left(p_{1}^{2} / q^{2}\right) p_{2 \mu}$ and $p_{2 \mu}^{\prime}=p_{2 \mu}-\left(p_{2}^{2} / q^{2}\right) p_{1 \mu}$ so that ${p^{\prime}}_{1}^{2}={p^{\prime}}_{2}^{2}=0,2 p^{\prime}{ }_{1} p^{\prime}{ }_{2}=q^{2}$. Additionally, $\alpha$ and $\beta$ are parameters, and $k_{\perp \mu}$ is the component of $k_{\mu}$ orthogonal to both $p_{1}$ and $p_{2}$.

Making use of the Sudakov parameterization, we can trace the origin of the separate terms for the one-loop form factor of Eq. (3). The first term $\left[\ln ^{2}\left(q^{2} / m^{2}\right)\right]$ arises from the integration over the region $1 \gg \alpha, \beta \gg m^{2} / q^{2}$ and $\alpha \beta \gg m^{2} / q^{2}$ for the case of an on-shell electron. When the initial (final) electron is off-shell, the condition $\alpha \gg p_{1}^{2} / q^{2}$ $\left(\beta \gg p_{2}^{2} / q^{2}\right)$ applies (provided the virtualities of the electron are so small that $p_{1}^{2} p_{2}^{2}<m^{2} q^{2}$ ) and we obtain the second $\left[-\ln ^{2}\left(p_{1}^{2} / m^{2}\right)\right]$ and the third $\left[-\ln ^{2}\left(p_{2}^{2} / m^{2}\right)\right]$ DL terms of Eq. (3). Finally, when $p_{1}^{2} p_{2}^{2}>m^{2} q^{2}$, the integration region is $1 \gg \alpha \gg p_{1}^{2} / q^{2}, 1 \gg \beta \gg p_{2}^{2} / q^{2}$, and this leads to the last term $\left[\Theta\left(p_{1}^{2} p_{2}^{2}-m^{2} q^{2}\right) \ln ^{2}\left(m^{2} q^{2} / p_{1}^{2} p_{2}^{2}\right)\right]$ of Eq. (3).

When considering only the first three terms of Eq. (3), the off-shell $W$ and on-shell $W$ are related analytically. One can easily take the limit $p_{1}^{2}, p_{2}^{2} \rightarrow m^{2}$ in the off-shell $W$ to obtain the on-shell $W$. However, the last term $\left[\Theta\left(p_{1}^{2} p_{2}^{2}-m^{2} q^{2}\right) \ln ^{2}\left(m^{2} q^{2} / p_{1}^{2} p_{2}^{2}\right)\right]$ of Eq. (3) changes this situation and results in a non-trivial relation between the on-shell and off-shell form factors.

Beyond QED, problems relating $S\left(q^{2}\right)_{o n}$ and $S\left(q^{2}\right)_{o f f}$ also exist in QCD and in the EW Standard Model. Additionally, when calculating DL scattering amplitudes in the EW Standard Model at energies $\gg 100 \mathrm{GeV}^{2}$, the situation becomes more involved because, in addition to the virtual photon exchanges, one must include $W$ and $Z$ exchanges. In contrast to QED, the logarithmic contributions from the $W$ and $Z$ bosons are regulated by their masses $\left(M_{W}\right.$ and $M_{Z}$ ); therefore, one should not introduce an infrared cut-off for them.

To simplify our notation, we introduce the mass scale $M$

$$
M \approx M_{Z} \approx M_{W},
$$

which we will use to treat the $W$ and $Z$ exchange processes in a more symmetric manner. We stress that introducing $M$ is purely a technical point, which is useful for performing an all-order summations of DL contributions in the Standard Model.

Most of the available DL calculations of EW on-shell amplitudes have been performed in the one-loop and two-loop approximation in the EW couplings, [4, 5] An all-orders DL summation for the EW on-shell amplitudes is also wellstudied for the case of the hard kinematics where the total resummation of DL contributions exponentiates [6, [8]. The more complicated case of the Regge kinematics was studied in detail in Refs. [10].

In the present paper, we relate the off-shell and on-shell double-logarithmic scattering amplitudes in QED, QCD, and the EW Standard Model. We obtain explicit expressions for the off-shell double-logarithmic scattering amplitudes in the Feynman gauge (as the off-shell amplitudes are gauge-dependent), and we also discuss the Coulomb gauge.

The organization of the paper is as follows. In Sect. 2 we relate the double-logarithmic off-shell and on-shell amplitudes in QED in the hard kinematic region. In Sec. 3 we consider more involved examples of the off-shell $2 \rightarrow 2$ scattering amplitudes where both virtual photons and $W, Z$ bosons are accounted to all orders in the electroweak couplings. In Sect. 4 we generalize the technique of Sect. 2 to the case of the forward Regge kinematics, and obtain explicit expressions for the DIS non-singlet structure function $g_{1}$ with the initial quark being off-shell. Specifically, we examine the effect of the initial quark virtuality on the small- $x$ asymptotics of $g_{1}$. Finally, in Sect. 5 we present our conclusions. 


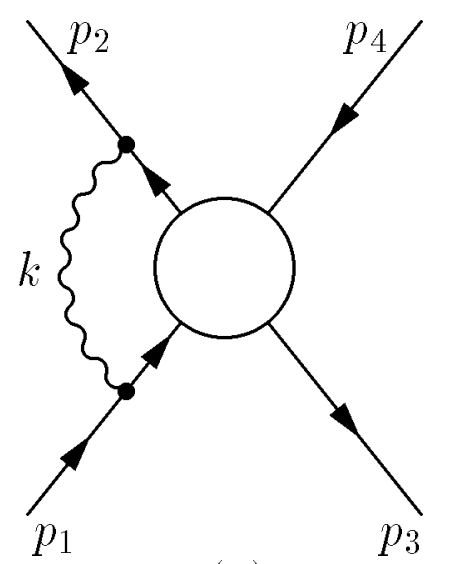

(a)

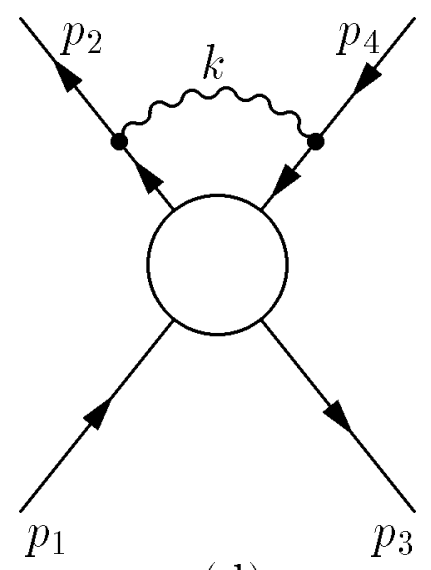

(d)

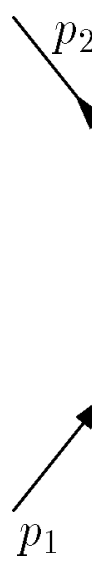

$p_{1}$

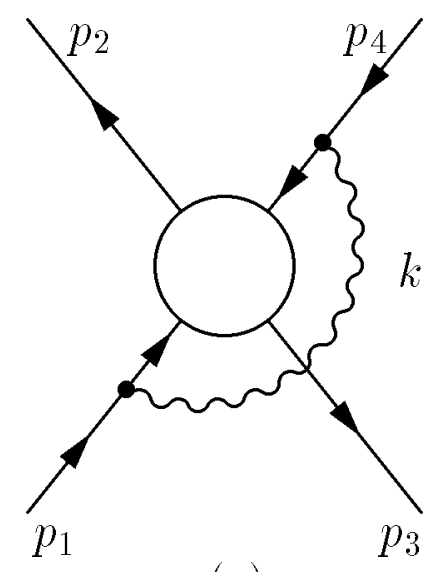

(e)

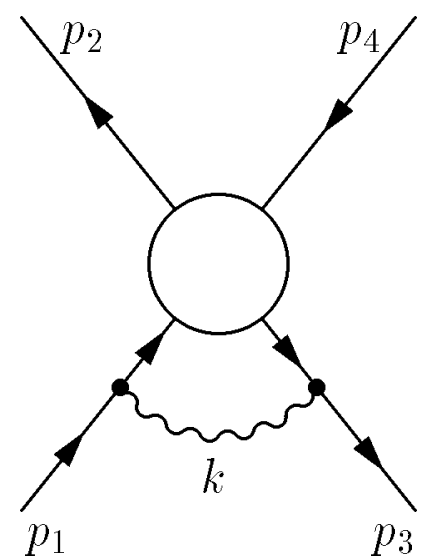

(c)

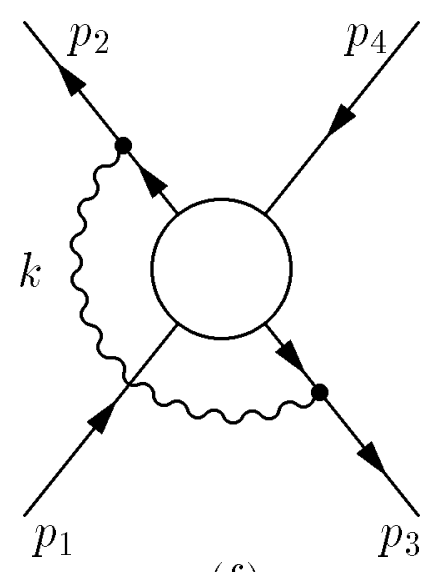

(f)

Figure 1: Diagrams showing the factorization of the softest photon with momentum $k$ when the Feynman gauge is used.

\section{RELATIONS BETWEEN ON-SHELL AND OFF-SHELL AMPLITUDES IN QED}

In the present section, we obtain a general prescription for relating off-shell and on-shell scattering amplitudes in the DLA for the (simplest) case of QED. We consider a $2 \rightarrow 2$ process in QED, where the initial and final particles are fermions. Although we derive our results for a general process, we will use the $e^{+} e^{-}$annihilation into quark or lepton pairs [e.g., $e^{-}\left(p_{1}\right) e^{+}\left(p_{2}\right) \rightarrow q^{-}\left(p_{3}\right) q^{+}\left(p_{4}\right)$ ] as our "model" process; the final formula however are valid for any channel. For this $2 \rightarrow 2$ process, we work in the hard kinematic limit where:

$$
s=\left(p_{1}+p_{2}\right)^{2} \sim-t=-\left(p_{3}-p_{1}\right)^{2} \sim-u=\left(p_{1}-p_{4}\right)^{2} \gg\left|p_{1,2}^{2}\right|,\left|p_{3,4}^{2}\right| .
$$

We will use the Infrared Evolution Equations (IREE) to resum the DL contributions. ${ }^{2}$ The IREE method has successfully been used to calculate the scattering amplitudes of various processes in QED, QCD and the EW Standard Model. $^{3}$ The first step is to use the IREE to factorize the DL contributions from the "softest" virtual particle, which we define to be the particle with the minimal transverse momentum $k_{\perp}$.

For QED scattering amplitudes in the hard kinematic limit Eq. (6), the softest particles must be photons. Their DL

\footnotetext{
${ }^{2}$ For a recent alternate approach, see Refs. 7, 8, 9].

${ }^{3}$ See Refs. [3, 6, 10, 12, 13, 15], and the references therein.
} 


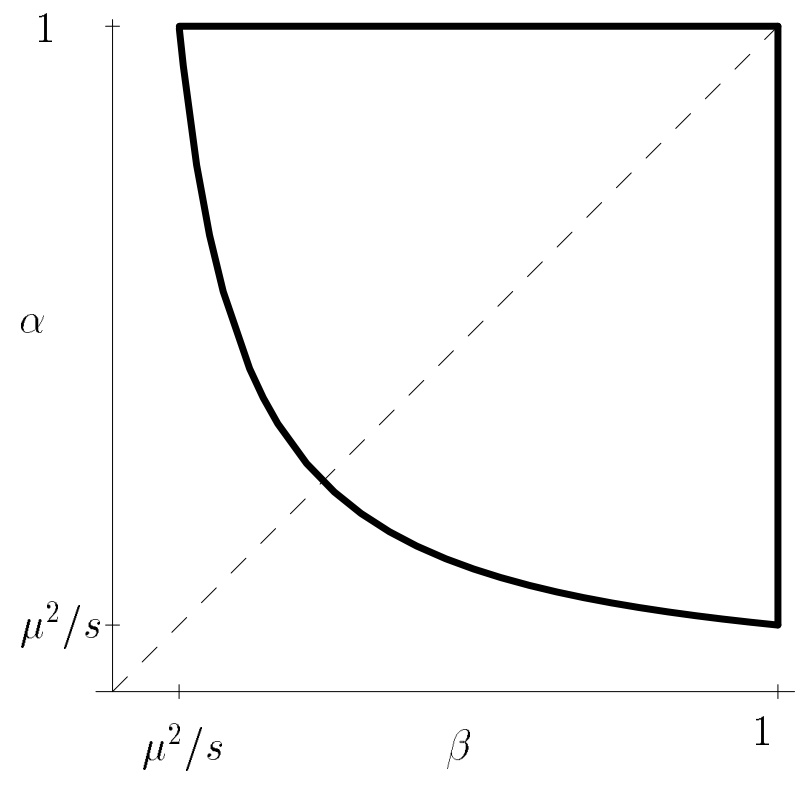

(a)

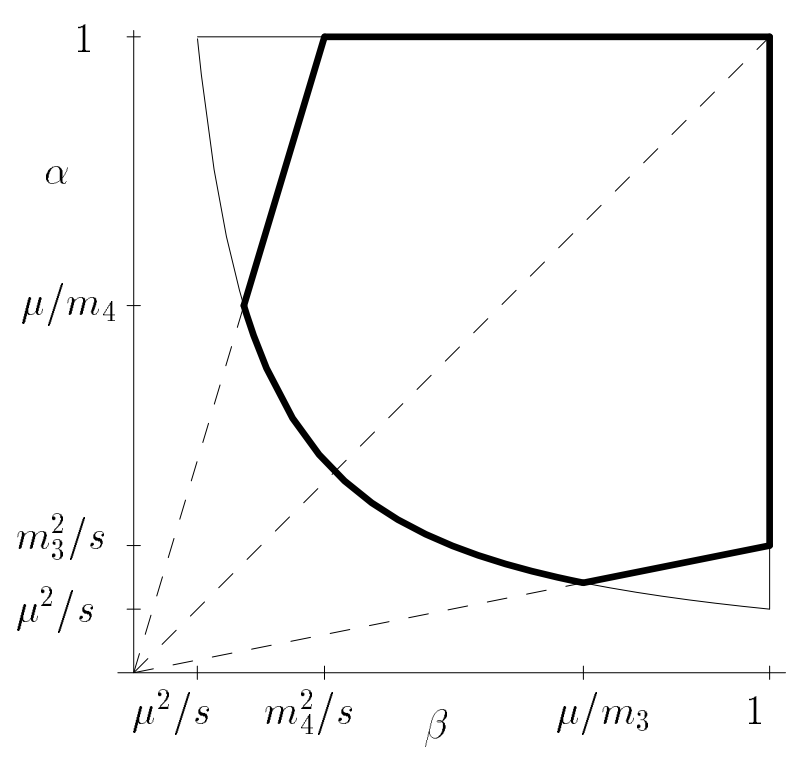

(b)

Figure 2: The integration region over the softest photon momentum when all external particles are on-shell and the infrared cut-off $\mu$ is chosen (a) as in Eq. [8), and (b) as in Eq. [16].

contributions can be factorized according to the Gribov bremsstrahlung theorem. ${ }^{4}$ The integration region over the softest photon momentum contains an infrared singularity, and therefore we must introduce an infrared cut-off, $\mu$. We impose this cut-off in the transverse momentum space by restricting the transverse momenta of all virtual particles to be greater than $\mu$. This constraint modifies the boundaries of the integration region, and results in relations between the cut-off scale $\mu$ and the masses (virtualities) of the external particles.

\section{A. The case of the on-shell amplitude $A\left(s, \mu^{2}\right)$ with massless external particles}

We begin with the well-know case where all the external particles are on-shell:

$$
p_{1,2}^{2}=m_{1,2}^{2}, \quad p_{3,4}^{2}=m_{3,4}^{2} \text {. }
$$

For simplicity, we take the case where the fermion masses are negligible:

$$
\mu^{2}>m_{1,2}^{2}, m_{3,4}^{2} \text {. }
$$

For this case, in the hard kinematic region the amplitude $A$ only depends on $s$ and $\mu^{2}$, and we can easily obtain the IREE for this process. The RHS of the IREE includes the Born term, $A^{\text {Born }}$, and the contributions of the graphs depicted in Fig. 1. These graphs represent all the possible ways to factorize the softest photon, providing the Feynman gauge is used. Using the standard Feynman rules, we obtain at the following IREE:

$$
A\left(s, \mu^{2}\right)=A^{\text {Born }}-2 \lambda Q^{2} \int_{\mu^{2}}^{s} \frac{d k_{\perp}^{2}}{k_{\perp}^{2}} \ln \left(s / k_{\perp}^{2}\right) A\left(s, k_{\perp}^{2}\right),
$$

where $\lambda=\alpha / 8 \pi$, and $Q$ is the modulus of the electric charge of the fermions. In Eq. (9) recall that $k_{\perp}$ plays the role of a new cut-off for any other virtual particles inside the blobs in Fig. 1,

\footnotetext{
${ }^{4}$ The generalization of Gribov bremsstrahlung theorem [11] to QCD discussed in Refs. 12, 13, 14].
} 
The logarithmic factor in the integrand of Eq. (9) arises from the integration over the longitudinal Sudakov variable $\beta$ [see Eq. [4]] of the factorized photon in the region $\mu^{2} \ll k_{\perp}^{2} \ll s \beta \ll s$. The integration region over $\beta$ and $k_{\perp}^{2}$ is especially simple in terms of the variables $\beta$ and $\alpha \equiv k_{\perp}^{2} / s \beta$, and this region is depicted in Fig. 2a. Differentiation with respect to $\ln \mu^{2}$ converts the integral equation Eq. (9) into a differential one:

$$
\partial A(x) / \partial x=-2 \lambda Q^{2} x A(x)
$$

with the solution

$$
A\left(s, \mu^{2}\right)=A^{B} \exp \left(-\lambda Q^{2} x^{2}\right) .
$$

Here, we have introduced the following notations: $x=\ln \left(s / \mu^{2}\right), \lambda=\alpha /(8 \pi)$, and $Q^{2}$ is defined to be:

$$
Q^{2}=Q_{1}^{2}+Q_{2}^{2}+Q_{3}^{2}+Q_{4}^{2},
$$

with $Q_{i}(i=1,2,3,4)$ being the modulus of the electric charge of the initial and final particles in units of $e=\sqrt{4 \pi \alpha}$. As we are using the Feynman gauge, the DL contributions from the graphs depicted in Fig. 1 yield another constraint for $Q^{2}$ :

$$
Q^{2}=Z_{a}+Z_{b}+Z_{c}+Z_{d}-Z_{e}-Z_{f}
$$

where the subscripts $a, . ., f$ refer to the graphs $a, . ., f$ in Fig. 1. Furthermore, we have the additional relations

$$
\begin{aligned}
Z_{a} & \equiv 2 Q_{1} Q_{2} \\
Z_{b} & \equiv 2 Q_{3} Q_{4} \\
Z_{c} & \equiv 2 Q_{1} Q_{3} \\
Z_{d} & \equiv 2 Q_{2} Q_{4} \\
Z_{e} & \equiv 2 Q_{1} Q_{4} \\
Z_{f} & \equiv 2 Q_{2} Q_{3} .
\end{aligned}
$$

Finally, making use of electric charge conservation ${ }^{5}$

$$
Q_{1}-Q_{2}-Q_{3}+Q_{4}=0
$$

we can rewrite $Q^{2}$ in the form of Eq. (12).

\section{B. The case of the on-shell amplitude $\tilde{A}\left(x, y_{1}, y_{2}\right)$ with massive external particles}

When $\mu$ is less than some of the masses of the external particles, the situation becomes more complex. We focus here on the specific case where:

$$
m_{1,2}<\mu<m_{3,4} .
$$

In this case, the scattering amplitude $\tilde{A}$ depends on $m_{3,4}^{2}$ in addition to $s$ and $\mu^{2}$. The integration region in the $\{\alpha, \beta\}$ plane $^{6}$ is depicted in Fig. 2b; note how this differs from the integration region for Eq. (9) shown in Fig. 2a. Performing the $\beta$ integration over the region of Fig. $2 \mathrm{~b}$, and differentiating the remaining integral with respect to $\mu$, we arrive at the new IREE in the differential form:

$$
\partial \tilde{A} / \partial x+\partial \tilde{A} / \partial y_{1}+\partial \tilde{A} / \partial y_{2}=-2 \lambda\left(Q^{2} x-Z_{A} y_{1} / 2-Z_{B} y_{2} / 2\right) \tilde{A}
$$

with $y_{1,2}=\ln \left(m_{3,4}^{2} / \mu^{2}\right), Z_{A}=\left(Z_{b}+Z_{c}-Z_{f}\right)=2 Q_{3}^{2}$, and $Z_{B}=Z_{b}+Z_{d}-Z_{e}=2 Q_{4}^{2}$. The general solution to Eq. (17) is

\footnotetext{
${ }^{5}$ In the explicit formula of this paper we refer to the annihilation channel, although the final results are true in any channel.

${ }^{6}$ Recall $\alpha \equiv k_{\perp}^{2} / s \beta$, and $s \beta$ is the longitudinal momentum of the softest photon.
} 


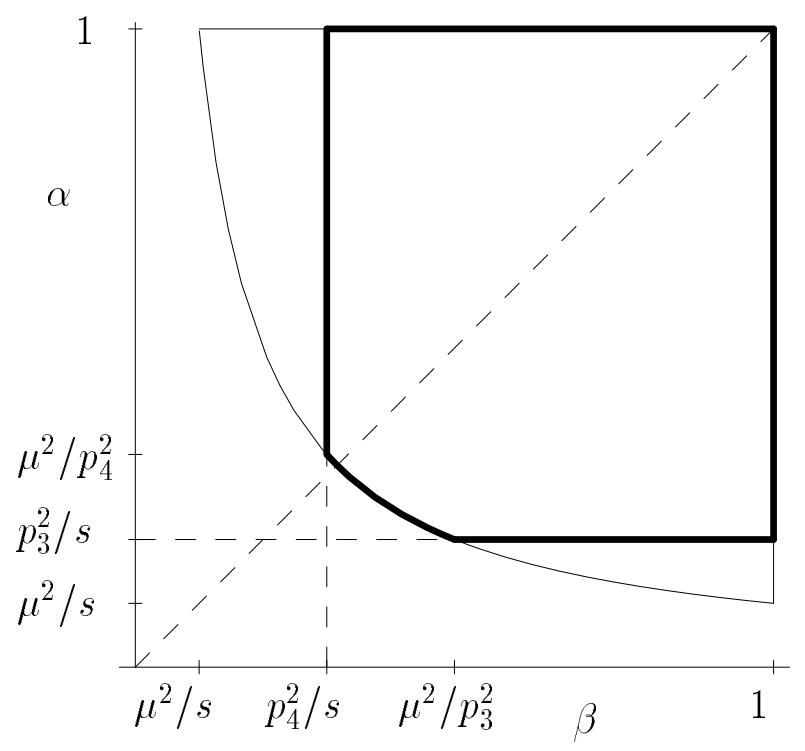

(a)

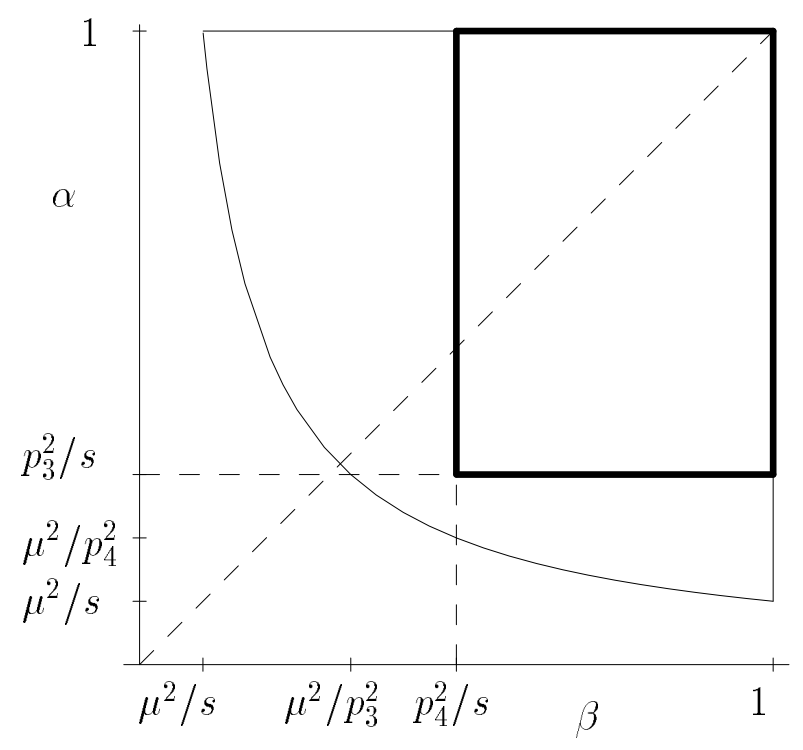

(b)

Figure 3: The integration region over the softest photon momentum when the final particles are (a) moderately-virtual and (b) deeply-virtual.

$$
\tilde{A}\left(x, y_{1}, y_{2}\right)=\tilde{\Phi}\left(x-y_{2}, y_{1}-y_{2}\right) \exp \left[-\lambda y_{2}\left(2 Q^{2} x-2 Z_{A} y_{1}+\left(-Q^{2}+Z_{A}-Z_{B}\right) y_{2}\right)\right]
$$

For $y_{2}=\ln \left(m_{4}^{2} / \mu^{2}\right)=0$ (i.e., $\left.m_{4}^{2}=\mu^{2}\right)$ there should be an obvious matching condition

$$
\left.\tilde{A}\left(x, y_{1}, y_{2}\right)\right|_{y_{2}=0}=\tilde{A}^{\prime}\left(x, y_{1}\right),
$$

where $\tilde{A}^{\prime}\left(x, y_{1}\right)$ is the amplitude of the same process, but for the case where $m_{1,2}<m_{4} \lesssim \mu<m_{3}$. This matching allows us to fix the unknown function $\tilde{\Phi}\left(x-y_{2}, y_{1}-y_{2}\right)$, providing the amplitude $\tilde{A}^{\prime}\left(x, y_{1}\right)$ is known. Taking the $y_{2}=0$ limit of Eq. (17), it is easy to see that $\tilde{A}^{\prime}\left(x, y_{1}\right)$ obeys the following IREE:

$$
\partial \tilde{A}^{\prime} / \partial x+\partial \tilde{A}^{\prime} / \partial y_{1}=-2 \lambda\left(Q^{2} x-Z_{A} y_{1} / 2\right) \tilde{A}
$$

with the general solution

$$
\tilde{A}^{\prime}\left(x, y_{1}\right)=\tilde{\Phi}^{\prime}\left(x-y_{1}\right) \exp \left[-\lambda y_{1}\left(2 Q^{2} x-2 Z_{A} y_{1}+\left(-Q^{2}-Z_{A}\right) y_{1}\right)\right] .
$$

In order to determine the function $\tilde{\Phi}^{\prime}\left(x-y_{1}\right)$, we again apply the matching condition for $y_{1}=\ln \left(m_{3}^{2} / \mu^{2}\right)=0$ $\left(m_{3}^{2}=\mu^{2}\right)$

$$
\left.\tilde{A}^{\prime}\left(x, y_{1}\right)\right|_{y_{1}=0}=A(x)
$$

where the amplitude $A(x)$ is defined in Eq. (11). The matching condition of Eq. (22) immediately leads to the solution for $\tilde{A}^{\prime}\left(x, y_{1}\right)$ :

$$
\tilde{A}^{\prime}\left(x, y_{1}\right)=A\left(x-y_{1}\right) \exp \left[-\lambda y_{1}\left(2 Q^{2} x-2 Z_{A} y_{1}+\left(-Q^{2}-Z_{A}\right) y_{1}\right)\right] .
$$

Inserting this result in Eq. (19), we can obtain $\tilde{A}\left(x, y_{1}, y_{2}\right)$ in terms of $\tilde{A}^{\prime}\left(x, y_{1}\right)$

$$
\tilde{A}\left(x, y_{1}, y_{2}\right)=\tilde{A}^{\prime}\left(x-y_{2}, y_{1}-y_{2}\right) \exp \left[-\lambda y_{2}\left(2 Q^{2} x-2 Z_{A} y_{1}+\left(-Q^{2}+Z_{A}-Z_{B}\right) y_{2}\right)\right] .
$$

Finally, after performing all the substitutions, we find:

$$
\tilde{A}\left(x, y_{1}, y_{2}\right)=A^{B o r n} \exp \left[-\lambda\left(Q^{2} x^{2}-Z_{A} y_{1}^{2} / 2-Z_{B} y_{2}^{2} / 2\right)\right] .
$$

In general, one can use Eq. (19) when $m_{3}>m_{4}$. 


\section{The case of $A_{1}\left(s, \mu^{2}, p_{3}^{2}\right)$ with one off-shell final-state particle}

Let us consider now the situation when one of the final-state particles is off-shell:

$$
s \gg p_{3}^{2} \gg \mu^{2} .
$$

We denote the amplitude for this case as $A_{1}\left(s, \mu^{2}, p_{3}^{2}\right)$. Let us replace this by: In the DLA, this amplitude does not depend on $m_{3}$; therefore $m_{3}$ can be dropped from Eq. (8) and Eq. (16) relating the value of the cut-off and the fermion masses. However, in order to use the matching between the off-shell and on-shell amplitudes in the simplest manner, we will assume that $\mu$ obeys Eq. (8). In contrast to the case of the on-shell amplitudes, the softest photon can now be factorized out of $A_{1}\left(s, \mu^{2}, p_{3}^{2}\right)$ when $\mu^{2} \ll k_{\perp}^{2} \ll p_{3}^{2}$. As was observed in the previous case, the $\beta$-integration region of the longitudinal momentum $s \beta$ of the softest photon momentum is now different for each individual graph in Fig. 1. Specifically, when the propagator of the photon connects to an on-shell external line, the integration region (depicted in Fig. 2a) yields the factor $\ln \left(s / k_{\perp}^{2}\right)$. Conversely, when the off-shell line with momentum $p_{3}$ is involved, the integration region (depicted in Fig. 3a) yields instead the factor $\ln \left(s / p_{3}^{2}\right)$. This leads to the following integral form for the IREE:

$$
\begin{aligned}
A_{1}\left(s / \mu^{2}, p^{\prime 2} / \mu^{2}\right) & =A_{1}^{B o r n}-2 \lambda\left[Z_{C} \int_{\mu^{2}}^{p^{\prime 2}} \frac{d k_{\perp}^{2}}{k_{\perp}^{2}} \ln \left(s / k_{\perp}^{2}\right) A_{1}\left(s / k_{\perp}^{2}, p_{3}^{2} / k_{\perp}^{2}\right)\right. \\
& \left.=+Z_{A} \int_{\mu^{2}}^{p^{\prime 2}} \frac{d k_{\perp}^{2}}{k_{\perp}^{2}} \ln \left(s / p_{3}^{2}\right) A_{1}\left(s / k_{\perp}^{2}, p_{3}^{2} / k_{\perp}^{2}\right)\right]
\end{aligned}
$$

Here, we have defined $Z_{C}=Q^{2}-Z_{A}$ where $Z_{A}$ was introduced in Eq. (17). Differentiating Eq. (27) with respect to $\mu^{2}$, we obtain a partial differential equation for $A_{1}\left(s, \mu^{2}, p_{3}^{2}\right)$

$$
\partial A_{1} / \partial x+\partial A_{1} / \partial z_{1}=-2 \lambda\left(Q^{2} x-Z_{A} z_{1}\right) A_{1}
$$

where we have introduced the new variable $z_{1}=\ln \left(p_{3}^{2} / \mu^{2}\right)$. The solution to Eq. 28) is given by

$$
\left.A_{1}\left(x, z_{1}\right)=\Phi\left(x-z_{1}\right) \exp \left[-2 \lambda Q^{2}\left(x-z_{1}\right) z_{1}+\lambda Z_{A} z_{1}^{2}\right)\right]
$$

where the function $\Phi$ is, as yet, unknown. In order to determine $\Phi$, we use the matching conditions at $z_{1}=\ln \left(p_{3}^{2} / \mu^{2}\right)=$ 0 (i.e., $p_{3}^{2}=\mu^{2}$ )

$$
\left.A_{1}\left(x, z_{1}\right)\right|_{z_{1}=0}=A(x)
$$

With this relation, we then obtain

$$
\left.A_{1}\left(x, z_{1}\right)=A\left(x-z_{1}\right) \exp \left[-2 \lambda Q^{2}\left(x-z_{1}\right) z_{1}-\lambda Z_{C} z_{1}^{2}\right)\right]
$$

which relates the off-shell amplitude $A_{1}$ to the on-shell amplitude $A$. Using Eq. (11), we obtain an explicit expression for $A_{1}\left(x, z_{1}\right)$ :

$$
A_{1}\left(x, z_{1}\right)=A^{\text {Born }} \exp \left[-\lambda\left(Q^{2} x^{2}-Z_{A} z_{1}^{2}\right)\right]
$$

\section{The case of $A_{2}\left(s, p_{3}^{2}, p_{4}^{2}, \mu^{2}\right)$ and $\widetilde{A}_{2}\left(x, y_{1}, y_{2}\right)$ with two off-shell final-state particles}

Let us consider now the amplitude $A_{2}\left(s, p_{3}^{2}, p_{4}^{2}, \mu^{2}\right)$ where both the final-state particles are off-shell. The amplitude $A_{2}\left(s, p_{3}^{2}, p_{4}^{2}, \mu^{2}\right)$ will obey different IREE depending on the value of the virtualities; we will address these in turn. 
1. Moderately-Virtual Kinematics: $A_{2}\left(s, p_{3}^{2}, p_{4}^{2}, \mu^{2}\right)$

We first consider the case where $p_{3,4}^{2}>\mu^{2}$ but with the condition

$$
p_{3}^{2} p_{4}^{2}<s \mu^{2} .
$$

We will refer to this as the moderately-virtual case. The IREE for $A_{2}\left(x, z_{1}, z_{2}\right)$ is similar to Eq. (28):

$$
\partial A_{2} / \partial x+\partial A_{2} / \partial z_{1}+\partial A_{2} / \partial z_{2}=-2 \lambda\left(Q^{2} x-Z_{A} z_{1}-Z_{B} z_{2}\right) A_{2},
$$

where we define $z_{1,2} \equiv \ln \left(p_{3,4}^{2} / \mu^{2}\right)$, and $Z_{A, B}$ is introduced in Eq. [17). Again, we solve this equation and using the matching conditions at $z_{2}=0$ (i.e., $p_{3}^{2}=\mu^{2}$ )

$$
\left.A_{2}\left(x, z_{1}, z_{2}\right)\right|_{z_{2}=0}=A_{1}\left(x, z_{1}\right)
$$

and eventually obtain

$$
A_{2}\left(x, z_{1}, z_{2}\right)=A^{B o r n} \exp \left[-\lambda\left(Q^{2} x^{2}-Z_{A} z_{1}^{2}-Z_{B} z_{2}^{2}\right)\right] .
$$

\section{Deeply-Virtual Kinematics: $\widetilde{A}_{2}\left(x, y_{1}, y_{2}\right)$}

Finally, let us consider the case of the deeply-virtual kinematics

$$
p_{3}^{2} p_{4}^{2}>s \mu^{2} .
$$

We denote the deeply-virtual amplitude as $\widetilde{A}_{2}\left(x, y_{1}, y_{2}\right)$. As the integration in graph (b) of Fig. 1 does not depend on $\mu$, the IREE is similar to that of Eq. (34), with the exception of the contribution proportional to $Z_{b}$ :

$$
\partial \tilde{A}_{2} / \partial x+\partial \tilde{A}_{2} / \partial z_{1}+\partial \tilde{A}_{2} / \partial z_{2}=-2 \lambda\left(\tilde{Q}^{2} x-\tilde{Z}_{A} z_{1}-\tilde{Z}_{B} z_{2}\right) \tilde{A}_{2}
$$

Here, the replacement $\tilde{Q}^{2}=Q^{2}-Z_{b}, \tilde{Z}_{A, B}=Z_{A, B}-Z_{b}$ should be made because the integration of graph (b) in Fig. 1 is performed over the region depicted in Fig. 3b. The solution of Eq. (38) is

$$
\tilde{A}_{2}\left(x, y_{1}, y_{2}\right)=\tilde{\Phi}_{2}\left(x-z_{1}, x-z_{2}\right) \exp \left[-\lambda\left(\tilde{Q}^{2} x^{2}-\tilde{Z}_{A} z_{1}^{2}-\tilde{Z}_{B} z_{2}^{2}\right)\right] .
$$

We determine the unknown function $\tilde{\Phi}_{2}$ using the matching condition at $p_{3}^{2} p_{4}^{2}=s \mu^{2}$ (i.e., when $x=z_{1}+z_{2}$ ), and obtain:

$$
\left.\tilde{A}_{2}\left(x, y_{1}, y_{2}\right)\right|_{x=z_{1}+z_{2}}=A_{2}\left(x, z_{1}, z_{2}\right) .
$$

For the function $\tilde{\Phi}_{2}$ we obtain

$$
\tilde{\Phi}\left(x-z_{1}, x-z_{2}\right)=\exp \left[-2 \lambda Z_{b}\left(x-z_{1}\right)\left(x-z_{2}\right)\right],
$$

and therefore the deeply-virtual amplitude $\tilde{A}_{2}\left(x, z_{1}, z_{2}\right)$ is given by:

$$
\begin{aligned}
\tilde{A}_{2}\left(x, z_{1}, z_{2}\right) & =A \exp \left[-\lambda\left[\tilde{Q}^{2} x^{2}-\tilde{Z}_{A} z_{1}^{2}-\tilde{Z}_{B} z_{2}^{2}+2 Z_{b}\left(x-z_{1}\right)\left(x-z_{2}\right)\right]\right] \\
& =A^{\text {Born }} \exp \left[-\lambda\left[Q^{2} x^{2}-Z_{A} z_{1}^{2}-Z_{B} z_{2}^{2}+Z_{b}\left(x-z_{1}-z_{2}\right)^{2}\right]\right] .
\end{aligned}
$$

The last term in the exponent of Eq. (42) contains the factor $Z_{b}$ defined by:

$$
Z_{b}=2 Q_{3} Q_{4}=Q_{3}^{2}+Q_{4}^{2}-\left(Q_{3}-Q_{4}\right)^{2} .
$$

Obviously, this factor cannot be rewritten in terms of the individual charges $Q_{3,4}^{2}$ of the external particles. 
When the Coulomb gauge is used for the on-shell amplitudes, DL contributions are always proportional to $Q_{k}^{2}$ because they come from only the self-energy graphs. [5, 16] Conversely, the interference graphs shown in Fig. 1 (i.e., the graphs where the boson propagators connect different external lines) do not yield DL contributions. This feature makes the Coulomb gauge especially convenient for the calculations of non-Abelian gauge theories; the nontrivial group factors are nothing but the standard Casimir factors. On the contrary, when the Feynman gauge is used, the DL contributions come from the interference graphs shown in Fig. 1, whereas the self-energy graphs do not yield DL contributions.

While this simple pattern holds true for on-shell amplitudes, it becomes more complex in the case of off-shell amplitudes. For off-shell amplitudes, both the Feynman and Coulomb gauges received DL contributions from the interference graphs. One can verify (using $\tilde{Q}^{2}=Z_{A}=Z_{B}=Z_{b}=2$ ) that Eqs. (11) and (42) reproduce, as a particular case, Eqs. (21) and (11) for the on-shell and off-shell Sudakov form factors of the electron. Finally, the generalization of these results to the case with more than 4 external particles can be calculated in the DLA in a similar manner.

\section{GENERALIZATION TO THE EW STANDARD MODEL}

When the $2 \rightarrow 2$ scattering amplitudes of Sect. 2 are considered in the framework of the ElectroWeak (EW) Standard Model, the situation becomes more involved due to introduction of two cut-off scales, $\mu$ and $M$. We will only comment briefly on the on-shell EW amplitudes in DLA, as these amplitudes have been computed in Refs. [6, 10] using the appropriate IREE's. We will examine in detail the $2 \rightarrow 2$ EW amplitudes in the hard kinematic limit.

In order to obtain the IREE for both photon and $W, Z$ exchanges, one must factorize the DL contributions where the EW bosons have minimal $k_{\perp}$. If the softest boson is a photon, then the lower limit of integration over $k_{\perp}$ is $\mu$, and the result resembles the QED case discussed in the previous section. Conversely, when the softest boson is a $W$ or $Z$, then the lower limit of integration over $k_{\perp}$ is $M$. Therefore, the integral on the RHS of Eq. (27) corresponding to the factorization of the softest photon should be replaced by the DL contributions of the factorization of the softest $W$ and $Z$ bosons. The contributions of the softest $Z$ boson looks quite similar to the photon case shown in Fig. 1; in contrast, the $W$ boson exchange changes the flavors of the interacting fermions.

Considerable technical difficulties arise when using either the Feynman or the Coulomb gauges. ${ }^{7}$ In the Coulomb gauge, the DL contributions of the softest bosons come from the boson self-energy graphs, and this means that the amplitudes are the exponential form obtained in Ref. [6]. On the contrary, when the Feynman gauge is used, the factorization of the softest $W$-boson leads to a system of four IREE's, the general solution of which consists of four exponentials. If one of these four exponentials were negative, this would lead to DL contributions that grow with $s$, and therefore violate unitarity. However, the Born contributions (which are the inhomogeneous terms in the integral IREE's) lead to the cancellation of three of the exponentials. The fourth exponential then coincides with the solution obtained using the Coulomb gauge. Consequently, the Coulomb gauge is more convenient for calculations in the hard kinematic limit of Eq. [6), but becomes inappropriate when working in the Regge kinematic region where the Feynman gauge is more convenient. Indeed, in the Feynman gauge one can avoid the above technical problems by expanding the EW amplitude into invariant ones, as was shown in Ref. [10].

\section{A. The Generalized IREE}

Let us consider a $2 \rightarrow 2$ electroweak scattering amplitude $A\left(s, p_{3}^{2}, p_{4}^{2}, \mu^{2}, M^{2}\right)$ in the hard kinematic limit of Eq. (6). We use the notation of the previous Section for the momenta of the external particles. We consider several cases assuming again that the initial particles are always on-shell, whereas the final particles can be either on-shell or off-shell. For any of these cases, the amplitude obeys the following integral IREE:

$$
\begin{aligned}
A\left(s, p_{3}^{2}, p_{4}^{2}, \mu^{2}, M^{2}\right)= & A^{\text {Born }}-\frac{U}{16 \pi^{2}} \int_{D} \frac{d k_{\perp}^{2}}{k_{\perp}^{2}} \frac{d \beta}{\beta} A\left(s, p_{3}^{2}, p_{4}^{2}, k_{\perp}^{2}, M^{2}\right) \\
& -\frac{g^{2} V}{16 \pi^{2}} \int_{D^{\prime}} \frac{d k_{\perp}^{2}}{k_{\perp}^{2}} \frac{d \beta}{\beta} A\left(s, p_{3}^{2}, p_{4}^{2}, k_{\perp}^{2}, k_{\perp}^{2}\right)
\end{aligned}
$$

\footnotetext{
${ }^{7}$ For DL calculations with the Coulomb gauge, see e.g. Refs. [5, 16].
} 
where $A^{\text {Born }}$ is the Born approximation for $A$, and $g=\sqrt{4 \pi \alpha} / \sin \theta_{W}$ is the $S U(2)$-coupling of the EW Standard Model. The factors $U$ and $V$ depend on the particular relations between the cut-offs and virtualities $p_{1,2}^{2}$; we will specify them below. The first (second) integral in Eq. (44) corresponds to the factorization of the softest photon (weak boson). The integration regions $D$ and $D^{\prime}$ also depend on the relations between the parameters $p_{3}^{2}, p_{4}^{2}, \mu^{2}$, and $M^{2}$. Generally, the solution to Eq. (44) can be written as

$$
A=A^{\text {Born }} \exp (-\psi)
$$

with appropriate exponents $\psi$. When both final-state particles are on-shell, the integration over $\beta$ yields the common factor $\ln \left(s / k_{\perp}^{2}\right)$ in both the $U$ and $V$ integrals of Eq. (44) even through the integration over $k_{\perp}^{2}$ runs from $\mu^{2}$ to $s$ in the first integral and from $M^{2}$ to $s$ in the second one. In a manner similar to Eq. (10), one obtains the on-shell factor $U_{\text {on }}=32 \pi^{2} \lambda Q^{2}$. The on-shell weak factor, $C_{W Z}$, is the sum of the factors $V_{i}$ :

$$
C_{W Z}=V_{1}+V_{2}+V_{3}+V_{4}
$$

where the subscripts 1 and 2 ( 3 and 4 ) refer to the initial-state particles with momenta $p_{1,2}$ (the final-state particles with momenta $p_{3,4}$ ). The factors $V_{i}$ are expressed in terms of the Weinberg angle $\theta_{W}$, the weak isospins $T_{i}^{2}\left(T_{i}^{2}=3 / 4\right.$ for a fermion), the hypercharges $Y_{i}$, and the electric charges $Q_{i}$ of the initial-state and final-state particles:

$$
V_{i}=\left[T_{i}^{2}+\left(Y_{i}^{2} / 4\right) \tan ^{2} \theta_{W}-Q_{i}^{2} \sin ^{2} \theta_{W}\right] .
$$

Differentiating Eq. (44) with respect to the cut-offs, we obtain the on-shell exponent $\psi \equiv \psi_{\text {on }}$ : [6]

$$
\psi_{\text {on }}=\alpha Q^{2} /\left(8 \pi^{2}\right) \ln ^{2}\left(s / \mu^{2}\right)+g^{2} /\left(32 \pi^{2}\right) C_{W Z} \ln ^{2}\left(s / M^{2}\right) .
$$

We recall that the photon double-logarithmic term $\ln ^{2}\left(s / \mu^{2}\right)$ in Eqs. [1148) is obtained under the assumption that the cut-off $\mu$ is greater than any mass involved.

In the case where the initial-state particles are light but the final-state particles are so heavy that Eq. (16) is satisfied, the term $Q^{2} \ln ^{2}\left(s / \mu^{2}\right)$ should be replaced by

$$
Q^{2} \ln ^{2}\left(s / \mu^{2}\right)-\left(Z_{A} / 2\right) \ln ^{2}\left(m_{1}^{2} / \mu^{2}\right)-\left(Z_{B} / 2\right) \ln ^{2}\left(m_{2}^{2} / \mu^{2}\right)
$$

in agreement with Eq. 25.

\section{B. The off-shell case:}

When the final-state particles are off-shell, the regions $D$ and $D^{\prime}$ in Eq. 44 are more complicated. We study below several interesting situations, denoting these as $R_{1,2,3,4}$.

\section{1. $R_{1}$ Case: final-state particles of small virtuality}

We call $R_{1}$ the simplest case when the virtualities of the final particles are relatively small:

$$
\begin{aligned}
\mu^{2} & <p_{3,4}^{2}<M^{2} \ll s, \\
p_{3}^{2} p_{4}^{2} & <s \mu^{2} .
\end{aligned}
$$

This case is quite similar to Eq. (33), so we immediately conclude that the exponent $\psi_{1}$ of Eq. (45) for this case is

$$
\psi_{1}=\frac{\alpha}{8 \pi}\left[Q^{2} \ln ^{2}\left(s / \mu^{2}\right)-Z_{A} \ln ^{2}\left(p_{3}^{2} / \mu^{2}\right)-Z_{B} \ln ^{2}\left(p_{4}^{2} / \mu^{2}\right)\right]+\frac{g^{2}}{32 \pi^{2}} C_{W Z} \ln ^{2}\left(s / M^{2}\right) .
$$

2. $R_{2}$ Case: Deeply virtual for photon, and on-shell for $W / Z$ boson

In the second case, $R_{2}$, the virtualities $p_{3,4}^{2}$ are larger and satisfy

$$
\begin{aligned}
\mu^{2} & <p_{3,4}^{2}<M^{2}, \\
s \mu^{2} & <p_{3}^{2} p_{4}^{2}<s M^{2} .
\end{aligned}
$$


This kinematics are deeply-virtual for the softest photon (with the solution given by Eq. (42)), but at the same time it is on-shell for the softest $W, Z$-bosons (with the solution given by Eq. (11)). Therefore the exponent $\psi_{2}$ for this case is

$$
\psi_{2}=\lambda\left[\tilde{Q}^{2} x^{2}-\tilde{Z}_{A} z_{1}^{2}-\tilde{Z}_{B} z_{2}^{2}+2 Z_{b}\left(x-z_{1}\right)\left(x-z_{2}\right)\right]+\frac{g^{2}}{32 \pi^{2}} C_{W Z} \ln ^{2}\left(s / M^{2}\right)
$$

\section{3. $R_{3}$ Case: Deeply virtual for photon, and moderately virtual for $W / Z$ boson}

The case $R_{3}$, defined as

$$
\begin{aligned}
p_{3,4}^{2} & >M^{2} \\
s & <p_{3}^{2} p_{4}^{2}<s M^{2},
\end{aligned}
$$

describes a situation which is deeply-virtual for the photon, and moderately-virtual for the $W, Z$-bosons. Combining the results of Eq. (36) and Eq. (42), we find the solution:

$$
\begin{aligned}
\psi_{3}= & \lambda\left[\widetilde{Q}^{2} x^{2}-\widetilde{Z}_{A} z_{1}^{2}-\widetilde{Z}_{B} z_{2}^{2}+2 Z_{b}\left(x-z_{1}\right)\left(x-z_{2}\right)\right] \\
& +\frac{g^{2}}{32 \pi^{2}}\left[C_{W Z} \ln ^{2}\left(s / M^{2}\right)-2 V_{3} \ln ^{2}\left(p_{3}^{2} / M^{2}\right)-2 V_{4} \ln ^{2}\left(p_{4}^{2} / M^{2}\right)\right] .
\end{aligned}
$$

\section{4. $\quad R_{4}$ Case: Deeply virtual for photon $W / Z$ boson}

Finally, when the momentum $p_{3,4}^{2}$ are so large that

$$
\begin{aligned}
p_{3,4}^{2} & >M^{2} \\
p_{3}^{2} p_{4}^{2} & >s M^{2},
\end{aligned}
$$

the situation is deeply-virtual for all of the softest electroweak bosons, and this case is equivalent to the scheme with a single cut-off $M$. The EW exponent $\psi_{4}$ for this case corresponds to the Eq. (42):

$$
\begin{aligned}
\psi_{4} & =\left[\lambda \widetilde{Q}^{2} \ln ^{2}\left(s / \mu^{2}\right)+g^{2} \widetilde{C}_{W Z} /\left(32 \pi^{2}\right) \ln ^{2}\left(s / M^{2}\right)\right] \\
& -\left[\lambda \widetilde{Z}_{A} \ln ^{2}\left(p_{3}^{2} / \mu^{2}\right)+g^{2} \widetilde{X}_{3} /\left(32 \pi^{2}\right) \ln ^{2}\left(p_{3}^{2} / M^{2}\right)\right] \\
& -\left[\lambda \widetilde{Z}_{B} \ln ^{2}\left(p_{4}^{2} / \mu^{2}\right)+g^{2} \widetilde{X}_{4} /\left(32 \pi^{2}\right) \ln ^{2}\left(p_{4}^{2} / M^{2}\right)\right] \\
& +2\left[\lambda Z_{b}+g^{2} X_{b} /\left(32 \pi^{2}\right)\right] \ln ^{2}\left(s / p_{3}^{2}\right) \ln ^{2}\left(s / p_{4}^{2}\right)
\end{aligned} .
$$

Here, $X_{3,4}=2 V_{3,4}-X_{b}$, and $X_{b}=1 / 2+\left(1 / \cos ^{2} \theta_{W}\right)\left[t_{3}^{(3)}-Q_{3} \sin ^{2} \theta_{W}\right]\left[t_{3}^{(4)}-Q_{4} \sin ^{2} \theta_{W}\right]$, where $Q_{3,4}$ are the electric charges and $t_{3}^{3,4}$ are the eigenvalues of the $S U(2)$-generator $T_{3}$ acting on the final-state particles 3 and 4 , respectively. It is convenient to rewrite $\psi_{4}$ in the following form:

$$
\begin{aligned}
\psi_{4} & =\left[\lambda Q^{2} \ln ^{2}\left(s / \mu^{2}\right)+g^{2} C_{W Z} /\left(32 \pi^{2}\right) \ln ^{2}\left(s / M^{2}\right)\right] \\
& -\left[\lambda Z_{A} \ln ^{2}\left(p_{3}^{2} / \mu^{2}\right)+g^{2} X_{3} /\left(32 \pi^{2}\right) \ln ^{2}\left(p_{3}^{2} / M^{2}\right)\right] \\
& -\left[\lambda \widetilde{Z}_{B} \ln ^{2}\left(p_{4}^{2} / \mu^{2}\right)+g^{2} X_{4} /\left(32 \pi^{2}\right) \ln ^{2}\left(p_{4}^{2} / M^{2}\right)\right] \\
& +\left[\lambda Z_{b}+g^{2} X_{b} /\left(32 \pi^{2}\right)\right] \ln ^{2}\left(s M^{2} / p_{3}^{2} p_{4}^{2}\right) .
\end{aligned}
$$

Eq. (45), together with the phases specified in Eqs. (5153555758, describe the $2 \rightarrow 2$ off-shell electroweak scattering amplitudes in the hard kinematic limit for several virtualities of the final-state particles. The above techniques can be generalized for other particular cases in a similar manner.

\section{OFF-SHELL AMPLITUDES IN THE REGGE KINEMATICS}

The evaluation of the DL contributions to the on-shell and off-shell scattering amplitudes in the hard kinematic limit (6) is relatively straightforward, and leads to the exponentiation of the one-loop DL contributions. When the scattering amplitudes are evaluated in the forward Regge limit

$$
s \approx-u \gg-t
$$


or backward Regge limit

$$
s \approx-t \gg-u
$$

the DL expressions are more complicated.

The $2 \rightarrow 2$ EW Regge amplitudes at TeV energies for electron-positron colliders have been discussed previously in Refs. [10]. In the following, we will consider one example of the off-shell Regge amplitudes in the QCD theory. We obtain a relation between on-shell and off-shell amplitudes for quark scattering, and use this result to calculate the non-singlet structure functions for Deep Inelastic Scattering when the initial quark is off-shell. In doing so, we make use of the expressions for the on-shell non-singlet structure functions obtained in Ref. [15] at small $x$. These expressions account for the DL and single-logarithmic (SL) contributions to all orders in the QCD coupling $\alpha_{s}$. Explicit expressions combining the DGLAP 1] expressions and our total resummation (DL and SL contributions) are obtained in Ref. [17]. These combined results are especially important in the region of small $x$, as the combination leads to the power-like asymptotic behavior $\left(\sim x^{-\omega_{0}}\right)$ of the structure functions when $x \rightarrow 0$, with intercept $\omega_{0} \approx 0.4$. We briefly summarize these on-shell results.

According to the Optical Theorem, it is possible to relate the on-shell non-singlet structure function $F^{( \pm)}\left(x, Q^{2}\right)$ to the imaginary part of the forward Compton amplitude $A^{( \pm)}\left(x, Q^{2}\right)$ with $-t \leq \mu^{2}$. It is convenient to present $A^{( \pm)}\left(x, Q^{2}\right)$ in the form of the Mellin integral:

$$
A^{( \pm)}\left(x, Q^{2}\right)=\int_{-\imath \infty}^{+\imath \infty} \frac{d \omega}{2 \pi \imath}\left(\frac{s}{\mu^{2}}\right)^{\omega} \xi^{( \pm)}(\omega) T^{( \pm)}\left(\omega, Q^{2}\right)
$$

with the signature factor $\xi^{( \pm)}(\omega)=-\left(e^{-\imath \pi \omega} \pm 1\right) / 2$, total energy $s=Q^{2} / x$, and the infrared cut-off $\mu$. The Mellin amplitude $T^{( \pm)}\left(\omega, Q^{2}\right)$ obeys the following IREE:

$$
\partial T^{( \pm)} / \partial y+\omega T^{( \pm)}=T^{( \pm)} H^{( \pm)}(\omega)
$$

with anomalous dimensions $H^{( \pm)}$, and $y=\ln \left(Q^{2} / \mu^{2}\right)$ so that $\mu^{2}$ is the starting point of the $Q^{2}$-evolution. $H^{( \pm)}$ include both double-logarithmic and the most important part of the single-logarithmic contributions. From Refs. [15] we have

$$
H^{( \pm)}=(1 / 2)\left[\omega-\sqrt{\omega^{2}-B^{ \pm}(\omega)}\right]
$$

where

$$
B^{( \pm)}(\omega)=\left[(1+\omega / 2) 4 \pi C_{F} A(\omega)+D^{( \pm)}(\omega)\right] /\left(2 \pi^{2}\right)
$$

In Eq. (64), $D^{( \pm)}(\omega)$ and $A(\omega)$ can be expressed in terms of $\rho=\ln (1 / x), b=\left(33-2 n_{f}\right) / 12 \pi$, and the color factors $C_{F}=4 / 3, N=3$ :

$$
\begin{gathered}
D^{( \pm)}(\omega)=\frac{2 C_{F}}{b^{2} N} \int_{0}^{\infty} d \eta e^{-\omega \eta} \ln \left(\frac{\rho+\eta}{\eta}\right)\left[\frac{\rho+\eta}{(\rho+\eta)^{2}+\pi^{2}} \mp \frac{1}{\eta}\right], \\
A(\omega)=\frac{1}{b}\left[\frac{\eta}{\eta^{2}+\pi^{2}}-\int_{0}^{\infty} \frac{d \rho e^{-\omega \rho}}{(\rho+\eta)^{2}+\pi^{2}}\right] .
\end{gathered}
$$

In Eq. (66),$A(\omega)$ is the Mellin transform of $\alpha_{s}\left(k^{2}\right)=1 /\left(b \ln \left(-k^{2} / \Lambda^{2}\right)\right)$ with time-like argument $k^{2}$. Solving Eq. (62) and introducing $F^{( \pm)}\left(x, Q^{2}\right)$,

$$
F^{( \pm)}\left(x, Q^{2}\right)=\frac{1}{\pi} \Im A^{( \pm)}\left(x, Q^{2}\right)
$$

we obtain

$$
F^{( \pm)}\left(x, Q^{2}\right)=\left(e_{q}^{2} / 2\right) \int_{-\imath \infty}^{+\imath \infty} \frac{d \omega}{2 \pi \imath}(1 / x)^{\omega} C^{( \pm)}(\omega) \delta q(\omega) \exp \left(H^{( \pm)}(\omega) y\right)
$$

Eq. (67) relates $F^{(+)}$and the forward amplitude $A^{(+)}$via the Optical theorem. In fact, $F^{(+)}$is the non-singlet contribution to the structure function $F_{1}$, and $F^{(-)}$is the non-singlet contribution to the polarized structure function 
$g_{1} . \delta q$ is the initial quark density, which is commonly determined from fitting the experimental data. The coefficient functions $C^{( \pm)}$can be expressed in terms of the anomalous dimensions $H^{( \pm)}$as:

$$
C^{( \pm)}=\frac{\omega}{\omega-H^{( \pm)}(\omega)}
$$

We now consider the case where the initial quark is off-shell with virtuality $p^{2}, 8$

$$
\mu^{2}<\left|p^{2}\right| \ll Q^{2}
$$

When the initial quark is off-shell, the forward Compton amplitude $\widetilde{A}^{( \pm)}$depends on $p^{2}$ as well: $\widetilde{A}^{( \pm)}=$ $\widetilde{A}^{( \pm)}\left(s, Q^{2}, p^{2}, \mu^{2}\right)$. As before, it is convenient to work with the Mellin off-shell amplitude $G\left(\omega, Q^{2}, p^{2}, \mu^{2}\right)$ which is related to $\widetilde{A}^{( \pm)}\left(s, Q^{2}, p^{2}, \mu^{2}\right)$ through the Mellin transform (61). The IREE for the off-shell amplitude $G\left(\omega, Q^{2}, p^{2}, \mu^{2}\right)$ is similar to the one for the on-shell amplitude $T^{( \pm)}$:

$$
\partial G^{( \pm)}(\omega, y, z) / \partial y+\partial G^{( \pm)}(\omega, y, z) / \partial z+\omega G^{( \pm)}(\omega, y, z)=T^{( \pm)}(\omega, y) h^{( \pm)}(\omega, z)
$$

where $z=\ln \left(p^{2} / \mu^{2}\right) . h^{( \pm)}(\omega, z)$ are new anomalous dimensions which can be found from the following IREE:

$$
\partial h^{( \pm)} / \partial z+\omega h^{( \pm)}=D^{( \pm)}+h^{( \pm)} H^{( \pm)}(\omega)
$$

with $D^{( \pm)}$defined in Eq. (64).

Solving Eq. (72), and using the matching conditions for $z=0$

$$
\left.h^{( \pm)}(\omega, z)\right|_{z=0}=H^{( \pm)}(\omega)
$$

we obtain

$$
h^{( \pm)}=\left[H^{( \pm)}+\int_{0}^{z} d u e^{\left(\omega-H^{( \pm)}\right) u} D^{( \pm)}(u)\right] e^{-\left(\omega-H^{( \pm)}\right) z} .
$$

Once the $h^{( \pm)}$are known, we can calculate $G^{( \pm)}$. Substituting $h^{( \pm)}$and $T^{( \pm)}$into Eq. (71) and solving, we obtain

$$
G^{( \pm)}(\omega, y, z)=T^{( \pm)}(\omega, 2 \eta) e^{-\omega z}+e^{-\omega l} \int_{\eta}^{l} d u e^{\omega u} T^{( \pm)}(\omega, u+\eta) h^{( \pm)}(u-\eta)
$$

where $l=(y+z) / 2, \eta=(y-z) / 2$. Eq. (75) gives $G^{( \pm)}(\omega, y, z)=T^{( \pm)}(\omega, y)$ when $z=0$.

To demonstrate a simple application of Eq. (75), let us estimate the effect of the virtuality $p^{2}$ of the initial quark on the small- $x$ asymptotics of the off-shell non-singlet structure functions $\widetilde{F}^{( \pm)}$. Given the results of Refs. [15], when $x \rightarrow 0$ the small- $x$ asymptotics of the on-shell structure functions $F^{( \pm)}$are Regge-like:

$$
F^{( \pm)}\left(x, Q^{2}\right) \sim e_{q}^{2} \delta q\left(\omega_{0}^{( \pm)}\right) c^{( \pm)} \xi^{\omega_{0}^{( \pm)}} / \ln ^{3 / 2} \xi
$$

where $\xi=\sqrt{Q^{2} /\left(x^{2} \mu^{2}\right)}, c^{( \pm)}=\left[2(1-q) /\left(\pi \sqrt{B^{( \pm)}}\right)\right]^{1 / 2}, q^{( \pm)}=d \sqrt{B^{( \pm)}} /\left.d \omega\right|_{\omega=\omega_{0}^{( \pm)}}$, and the intercepts are $\omega_{0}^{(+)}=0.38$ and $\omega_{0}^{(-)}=0.43$. To estimate the asymptotics of $\widetilde{F}^{( \pm)}\left(x, Q^{2}, p^{2}\right)$, we neglect $D^{( \pm)}$in Eq. (74) since the effect of $D^{( \pm)}$ on the small- $x$ behavior of $g_{1}$ is not large. 15] Performing the integration in Eq. (75) using the saddle-point method, we obtain at the following expression for the small- $x$ asymptotics of the off-shell structure functions $\widetilde{F}^{( \pm)}$:

$$
\widetilde{F}^{( \pm)}\left(x, Q^{2}, p^{2}\right) \approx F^{( \pm)}\left(x, Q^{2}\right) e^{-\omega_{0} z / 2}\left[1+\omega_{0}^{( \pm)} z / 2\right]
$$

\footnotetext{
8 As in the previous Sections, we drop the modulus sign in Eq. 70, keeping the notation $p^{2}$ instead of $\left|p^{2}\right|$.
} 
with $\omega_{0}^{(+)}=0.38$ and $\omega_{0}^{(-)}=0.43 .{ }^{9}$ In order to estimate the difference between the on-shell and the off-shell non-singlet structure functions, we define the deviation $R$ :

$$
R=\left(F^{( \pm)}-\widetilde{F}^{( \pm)}\right) / F^{( \pm)} \approx 1-e^{-0.2 \ln \left(p^{2} / \mu^{2}\right)}\left[1+0.2 \ln \left(p^{2} / \mu^{2}\right)\right]
$$

where we estimate $\mu$ to be $\approx 1 \mathrm{GeV}$. 15] We observe from Eq. (78) that $R$ increases with increasing $p^{2}$. By definition, $R=0$ when $p^{2}=1 \mathrm{GeV}^{2}$ and therefore it grows when $p^{2}$ becomes greater than $\mu^{2}$ (we remind that the notation $p^{2}$ actually denotes $\left|p^{2}\right|$ ). In particular, $R \approx 0.02$ for $\left|p^{2}\right|=3 \mathrm{GeV}^{2}$ and $R \approx 0.08$ for $\left|p^{2}\right|=10 \mathrm{GeV}^{2}$.

\section{CONCLUSIONS}

In this paper, we have presented an approach for calculating the off-shell scattering amplitudes in the doublelogarithmic (DL) approximation. We have considered, in particular, the case where the initial particles are on-shell, but some of the final particles can be off-shell. Technically, this approach is based on constructing the appropriate infrared evolution equations (IREE) for the off-shell amplitudes. The specific form of the solutions depends on the size of the virtualities of the final particles. The general strategy for obtaining the off-shell solutions was to use the matching conditions to relate amplitudes of the same process, but with smaller virtualities of the final particles. Such a procedure allows us to relate the amplitudes with $N$ off-shell particles to those with $N-1$ off-shell particles. Using this recursive relation, we can eventually relate the off-shell amplitudes to the on-shell amplitudes.

To demonstrate the utility of this approach, we have computed the scattering amplitudes in the hard kinematic limit of Eq. (6), where the DL corrections simply exponentiation the one-loop DL contribution. We have reproduced the well-known results for the $2 \rightarrow 2$ on-shell QED amplitudes of Eqs. (11125), and used these results to obtain explicit expressions for the amplitudes with one (Eq. (32)) and two (Eqs. (3642)) final particles off-shell. We have considered both cases of moderate and large virtualities for the final particles, and compared the results in the Feynman and Coulomb gauges. In particular, we have shown that both the self-energy and interference graphs yield DL contributions in the Coulomb gauge; this result is contrary to the on-shell case. This result leads to the conclusion that the Coulomb gauge does not have provide any technical advantage, compared to the Feynman gauge, for calculating the off-shell amplitudes.

In Sect. 3 we have computed the more complex case of the off-shell amplitudes for the $2 \rightarrow 2$ electroweak processes in the hard kinematic limit at energies $\gg 100 \mathrm{GeV}$. In addition to the virtual photon exchanges, we also took the exchanges of virtual $W$ and $Z$ bosons into account. The results of the DL contributions for these scattering amplitudes depends on the virtualities of the final particles; the results are presented in Eqs. (51535557).

Finally, we have studied the QCD forward Compton scattering amplitude in the Regge kinematic limit (Eqs. (59600) with both the photons and the quarks being off-shell. This result was used to calculate the DIS non-singlet structure functions with off-shell initial quarks (Eq. (75)). To estimate the difference between the off-shell and the on-shell structure functions, we compared their small-x asymptotics (Eqs. (76/77), and the result is presented in Eq. (78). The on-shell and off-shell asymptotic amplitudes differ at larger values of $p^{2}$; for example, this difference is $\approx 8 \%$ for $p^{2}=10 \mathrm{GeV}^{2}$. A more accurate estimate of the effect of the virtuality on the structure functions could be directly obtained from the explicit expression of Eq. (75), instead of using their asymptotic behavior of Eq. (77); this topic will be reserved for future study.

\section{ACKNOWLEDGMENT}

We are grateful to A. Barroso for useful discussions. The work is supported in part by grant RSGSS-1124.2003.2, U.S. DoE grant DE-FG03-95ER40908, and the Lightner-Sams Foundation.

[1] G. Altarelli and G. Parisi, Nucl. Phys.B126 (1977) 297; V.N. Gribov and L.N. Lipatov, Sov. J. Nucl. Phys. 15 (1972) 438; L.N.Lipatov, Sov. J. Nucl. Phys. 20 (1972) 95; Yu.L. Dokshitzer, Sov. Phys. JETP 46 (1977) 641.

[2] V.V. Sudakov. Sov. Phys. JETP 3(1956)65.

\footnotetext{
9 See Refs. 15. for details.
} 
[3] B.I. Ermolaev and S.I. Troyan. Nucl. Phys.B590(2000)521.

[4] B.I. Ermolaev. Yad. Fiz. 28(1085)1978; P. Ciafaloni and D. Comelli. Phys.Lett.B 476(2000)49; M. Hori, H. Kawamura and J. Kodaira. Phys.Lett.B 491(2000)275; M. Melles. Phys.Lett.B 495(200)81; A. Denner, M. Melles and S. Pozzorini. Nucl.Phys.B 662(2003)299.

[5] W. Beenakker and A. Werthenbach. Nucl.Phys.B 630(2002)275;

[6] V.S. Fadin, L.N. Lipatov, A.D. Martin, and M. Melles, Phys.Rev. D61 (2000) 094002.

[7] M. Ciafaloni, P. Ciafaloni, D. Comelli. Phys.Rev.Lett.88(2002)102001.

[8] J.H. Kuhn, A.A. Penin. hep-ph/9906545 J.H. Kuhn, A.A. Penin, and V.A. Smirnov. Eur. Phys. J.C. 17(2000)97.

[9] E. Bartos, E.A. Kuraev, I.O. Cherednikov. Phys.Lett.B593(2004)115.

[10] B.I. Ermolaev, M. Greco and S.I. Troyan. Phys.Rev.D67(014017)2003; B.I. Ermolaev, S.M. Oliveira and S.I. Troyan. Phys.Rev.D66(114018)2002; A. Barroso, B.I. Ermolaev, M. Greco S.M. Oliveira, and S.I. Troyan. Phys.Rev.D69(034012)2004.

[11] V.N. Gribov. Yad. Fiz. 5(1967)199.

[12] R. Kirschner and L.N. Lipatov. Nucl. Phys. B213(1983)122

[13] B.I Ermolaev, V.S. Fadin, L.N. Lipatov. Yad. Fiz. 45(1987)817; B.I. Ermolaev. Sov. J. Nucl. Phys. $47(841) 1988$.

[14] M. Chaichian and B. Ermolaev. Nucl. Phys.B451(1995)194.

[15] B.I. Ermolaev, M. Greco and S.I. Troyan. Nucl.Phys.B 594 (2001)71; ibid 571(2000)137; Phys.Lett.B579(321),2004.

[16] A.V. Smilga and M.I. Vysotsky. Nucl. Phys. B150(1979)173.

[17] B.I. Ermolaev, M. Greco and S.I. Troyan. hep-ph/0503019 